\title{
Effect of transplantation of olfactory ensheathing cell conditioned medium induced bone marrow stromal cells on rats with spinal cord injury
}

\author{
LINJIE FENG, HONGQUAN GAN, WENGUO ZHAO and YINGJIE LIU \\ Department of Orthopedics, North China University of Science and Technology \\ Affiliated Hospital, Tangshan, Hebei 063000, P.R. China
}

Received December 29, 2016; Accepted February 16, 2017

DOI: $10.3892 / \mathrm{mmr} .2017 .6811$

\begin{abstract}
Spinal cord injury is a serious threat to human health and various techniques have been deployed to ameliorate or cure its effects. Stem cells transplantation is one of the promising methods. The primary aim of the present study was to investigate the effect of the transplantation of olfactory ensheathing cell (OEC) conditioned medium-induced bone marrow stromal cells (BMSCs) on spinal cord injury. Rat spinal cord compression injury animal models were generated, and the rats divided into the following three groups: Group A, (control) Dulbecco's modified Eagle's medium-treated group; group B, normal BMSC-treated group; group C, OEC conditioned medium-induced BMSC-treated group. The animals were sacrificed at 2, 4 and 8 weeks following transplantation for hematoxylin and eosin staining, and fluorescence staining of neurofilament protein, growth associated protein-43 and neuron-specific nuclear protein. The cavity area of the spinal cord injury was significantly reduced at 2 and 4 weeks following transplantation in group $\mathrm{C}$, and a significant difference between the Basso, Beattie and Bresnahan score in group $\mathrm{C}$ and groups $\mathrm{A}$ and $\mathrm{B}$ was observed. Regenerated nerve fibers were observed in groups B and C; however, a greater number of regenerated nerve fibers were observed in group $\mathrm{C}$. BMSCs induced by OEC conditioned medium survived in vivo, significantly reduced the cavity area of spinal cord injury, promoted nerve fiber regeneration following spinal cord injury and facilitated recovery of motor function. The present study demonstrated a novel method to repair spinal cord injury by using induced BMSCs, with satisfactory results.
\end{abstract}

Correspondence to: Dr Yingjie Liu, Department of Orthopedics, North China University of Science and Technology Affiliated Hospital, 73 Jianshe South Road, Tangshan, Hebei 063000, P.R. China

E-mail: linjiefengdoc@163.com

Key words: spinal cord injury, bone marrow stromal cells, conditioned medium, transplantation, rats

\section{Introduction}

Spinal cord injury poses a serious threat to human health. Spinal cord injury that occurs as a result of an external force, leads to primary nerve tissue damage and the rapid occurrence of secondary injury due to the activation of complex cascade reactions, which prevent the successful repair of spinal cord injury (1-5). Surgical decompression treatment is used during the early stage of clinical treatment for spinal cord injury. If cell transplantation using neural stem cells (NSCs), olfactory ensheathing cells (OECs) and bone marrow stromal cells (BMSCs) were to be simultaneously combined with this method, the treatment may demonstrate beneficial effects, and may be useful in clinical practice. Previous studies investigating the pathophysiology of spinal cord injury have demonstrated that the formation of the cystic cavity following spinal cord injury is an important factor in the prevention of axonal regeneration $(6,7)$. Therefore, effective treatments aim to prevent or reduce the formation of the cystic cavity following spinal cord injury, improve the micro-environment of the spinal cord injury sites and protect the damaged nerve cells, which is conducive to axonal regeneration $(3,8,9)$. Cell transplantation using NSCs, OECs and BMSCs is considered to be one of the most promising methods for the treatment of spinal cord injury. If the transplanted cells survive, proliferate in the site of spinal cord injury and integrate in the host tissues, it may be possible to protect the damaged spinal cord tissue, promote axonal regeneration and enhance functional recovery $(1,8-11)$.

Bone marrow stromal cells (BMSCs) are easy to acquire and culture, proliferate well in vitro and possess clinical potential. In response to different inducing factors or environmental effects, BMSCs differentiate into neuron-like cells (12-16). Previous studies have demonstrated that BMSCs efficiently differentiate into neuron-like cells in the presence of factors secreted by OECs (17-19). BMSCs have been used in the treatment and repair of nervous system injuries, and were observed to promote axonal regeneration by varying degrees, which led to improved motor function $(3,4,19-21)$. The primary aim of the present study was to determine whether the application of transplanted OEC-induced BMSC neural-like cells increases the recovery of injured spinal cord function, improve 
cell survival in the harsh environment following spinal cord injury, and promotes axonal regeneration.

\section{Materials and methods}

Culture and purification of OECs and preparation of conditioned medium. A total of 62 adult male Sprague-Dawley rats (weight, 200-220 g; age, 6 weeks) were obtained from the Experimental Animal Center of North China University of Science and Technology (Tangshan, China). They were individually housed in clear cages in a controlled environment with constant temperature of $23.8^{\circ} \mathrm{C}$ and humidity of $50 \pm 10 \%$. The animal room was on a 12:12 h light:dark cycle and with food and water available ad libitum. The present study was performed in strict accordance with the recommendations outlined in the Guide for the Care and Use of Laboratory Animals (13). The animal use protocol was reviewed and approved by the Institutional Animal Care and Use Committee of North China University of Science and Technology. A rat ( $250 \mathrm{~g}$ ) was sacrificed by cervical dislocation and the olfactory bulbs were separated under sterile conditions, the pia mater was discarded, and the olfactory nerve layer and the olfactory bulb granular layer were separated. The granular layer was washed twice with D-Hank's buffer, placed in a $37^{\circ} \mathrm{C}$ incubator, and digested with $0.125 \%$ trypsin (Sigma-Aldrich; Merck KGaA, Darmstadt, Germany) for $25 \mathrm{~min}$. Digestion was terminated with a trypsin terminator $(0.2 \mathrm{mM} / \mathrm{l}$; Sigma-Aldrich; Merck $\mathrm{KGaA}$, Darmstadt, Germany) for $8 \mathrm{~min}$ at $37^{\circ} \mathrm{C}$ and the mixture was centrifuged at $71.6 \mathrm{x}$ g for $5 \mathrm{~min}$ at $37^{\circ} \mathrm{C}$, before it was washed once with serum-free Dulbecco's modified Eagle's medium (DMEM)/F12 medium (Sigma-Aldrich; Merck $\mathrm{KGaA})$. Finally, single cell suspensions were produced using DMEM/F12 medium containing 20\% fetal calf serum (FCS; Sigma-Aldrich; Merck KGaA), seeded in plastic culture flasks and cultured in an incubator at $37^{\circ} \mathrm{C}$ and $5 \% \mathrm{CO}_{2}$. In accordance with the modified Nash differential adherent method $(2,3)$, the cells were aspirated and transferred onto polylysine (Sigma-Aldrich; Merck KGaA)-coated plastic flasks following 18-20 h of culture. Cytarabine (Ara-C; Sigma-Aldrich; Merck $\mathrm{KGaA}$ ) was then added 18-20 h later. Cells were incubated with 3-5 $\mu \mathrm{M} / 1$ Ara-C for 24-48 h, based on the number of fibroblasts as counted using light microscopy. The medium was refreshed every 2-3 days, with one-third of the medium replaced in the first 6 days, followed by $50 \%$ of the medium thereafter. The replacement medium contained $100 \mathrm{ml} \mathrm{20 \%}$ FCS. Once the purified OECs had reached 70-80\% confluence, (following 9-12 days), the culture medium was discarded and the cells were washed with fresh medium (DMEM/F12 containing $15 \%$ FCS) twice before fresh medium was added. The culture supernatant was aspirated following $24 \mathrm{~h}$ and centrifuged at $670.8 \times \mathrm{g}$ for $20 \mathrm{~min}$ at $37^{\circ} \mathrm{C}$. The supernatant was then collected and used as OEC conditioned medium in subsequent experiments.

BMSC culture and induced labeling prior to transplantation. A rat (weight, $150 \mathrm{~g}$ ) was sacrificed by cervical dislocation, and the complete bilateral femur and tibia were obtained under sterile conditions. Both sides of the metaphysis were successively cut to expose the bone marrow cavity, and the cavity was washed with DMEM medium (Gibco; Thermo
Fisher Scientific, Inc., Waltham, MA, USA) 2-3 times with a $2 \mathrm{ml}$ syringe. Collected fluid was filtered with a 200 mesh filter, before an equal volume of $0.84 \% \mathrm{NH}_{4} \mathrm{Cl}$ solution was added (3). The mixture was centrifuged at $850 \mathrm{r} / \mathrm{min}$ for $10 \mathrm{~min}$ at $37^{\circ} \mathrm{C}$ and the supernatant was discarded. Cells were resuspended in DMEM/F12 (Gibco; Thermo Fisher Scientific, Inc.) containing $15 \% \mathrm{FCS}$, inoculated in culture flasks and placed into a humidified incubator at $37^{\circ} \mathrm{C}$ and $5 \% \mathrm{CO}_{2}$. The medium was refreshed following $72 \mathrm{~h}$, and then once every three days. When the cells had reached $\sim 80 \%$ confluence, they were digested with $0.25 \%$ trypsin (Sigma-Aldrich; Merck $\mathrm{KGaA}$ ) for $10 \mathrm{~min}$ at $37^{\circ} \mathrm{C}$ and subcultured. BMSCs were separated from monocytes and lymphocytes, as the latter cell types adhere to the bottom of the culture flask while BMSCs are less adherent and are removed more easily. The cells were cultured until passage 3 , at which point they were adherent and four culture flasks were selected at random. The medium in two flasks was replaced with OEC conditioned medium to induce the BMSCs for $48 \mathrm{~h}$, and all four flasks of cells were stained with $10 \mu \mathrm{g} / \mathrm{ml}$ Hoechst 33342 (Sigma-Aldrich; Merck $\mathrm{KGaA}$ ) for $30 \mathrm{~min}$ at $37^{\circ} \mathrm{C}$ in order to label cell nuclei prior to transplantation $(2,4)$. The cells were subsequently washed with 0.01 M phosphate-buffered saline (PBS) 3-5 times and digested with trypsin for $10 \mathrm{~min}$ at $37^{\circ} \mathrm{C}$. Cells were then collected by centrifugation at $80.8 \times \mathrm{g}$ for $5 \mathrm{~min}$ at $37^{\circ} \mathrm{C}$, and resuspended to a final concentration of $\sim 1 \times 10^{5} / \mu 1$ for further use.

Animal grouping, modeling, and cell transplantation. Healthy male Sprague-Dawley rats $(n=60)$ were anesthetized with $1 \%$ sodium pentobarbital $(30 \mathrm{mg} / \mathrm{kg})$ by intraperitoneal injection. Under anesthesia, the animals were fixed on to the spinal cord injury instrument. Conventional skin preparation was performed as follows: The area was disinfected and towels were positioned around the T8 segment. The dorsal skin was successively cut and the muscles were separated on both sides of the spinous process, which was successively opened. A T7-9 laminectomy was then performed under the operating microscope to expose the T8 segment of the spinal cord. Each rat underwent spinal cord compression injury with a plastic spinal cord compression plate with a thickness of $0.5 \mathrm{~mm}$ and a breadth of $2.8 \mathrm{~mm}$. The compression plate was attached to a copper rod giving a total weight of $20 \mathrm{~g}$ on the spinal cord. The compression plate was lowered down ventrally at a rate of $0.5 \mathrm{~mm} / \mathrm{min}$ to the bottom of the vertebral canal and remained there for $5 \mathrm{~min}$. Following the successful preparation of models, the animals were divided at random into groups A, B and C (20 rats/group), which were injected with DMEM, BMSCs and induced BMSCs, respectively, into the spinal cord injury sites. The injection methods were as follows: The spinal cord injury center sites were selected and a point for injection with a depth of $1.7 \mathrm{~mm}$ was selected. The injection volume was $2 \mu \mathrm{l}$ and the injection speed was $0.2 \mu \mathrm{l} / \mathrm{min}$, with the needle slowly drawn back. The needle was retained for 5 min following injection. The redundant cells adversely flowed along the needle passage, so the process did not aggravate the spinal cord injury. The fascia and skin were sutured following surgery, and postoperative artificial assistance for drainage was provided twice per day in the first week and then once per day thereafter. Postoperative conventional insulation, anti-infection (penicillin $4 \times 10^{6} \mathrm{U} /$ day for 3 days after surgery; 


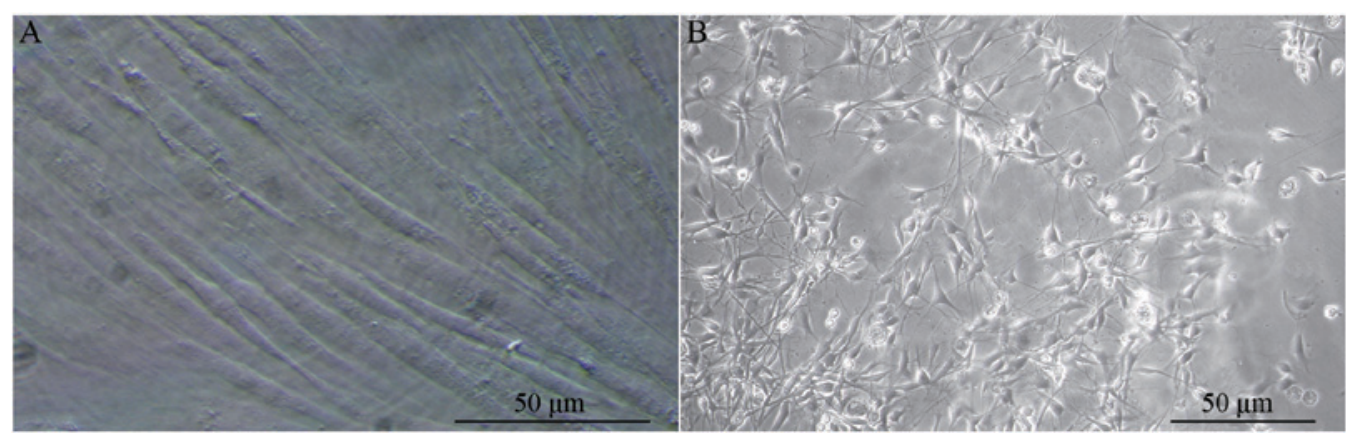

Figure 1. (A) Cultured bone marrow stromal cells at passage 3, which displayed fusiform growth (magnification, $\mathrm{x} 40$ ). (B) Primary culture olfactory ensheathing cells at day 9, which exhibited a bipolar spindle shape (magnification, $\mathrm{x} 40$ ).

Hoechst-Huabei Pharmaceuticals Co. Ltd., Shijiazhuang, China) and other paraplegia care were performed to prevent the formation of bedsores.

Evaluation of motor function in the lower extremities. Basso, Beattie and Bresnahan (BBB) ratings were used for rats in all groups, in order to assess motor function of the lower limbs (2-5), and the double-blind method was used for assessment. BBB scores range between 0 (complete paralysis) and 21 (normal). Early Stage (0-7): Isolated joint movements with little or no hind limb movement; Intermediate Stage (8-13): Intervals of uncoordinated walking; and, Late Stage (14-21): Fore and hind limb coordination. Each rat was assessed for at least 4 min once a week following injury for a total of 8 weeks.

Histological examination of the cavity area of spinal cord injury. At 2 weeks following surgery, 4 rats were randomly selected from each group. Following excess anesthesia (1\% sodium pentobarbital; Sigma-Aldrich; Merck KGaA) by intraperitoneal injection, specimens were fixed with $4 \%$ paraformaldehyde via the left ventricle using conventional perfusion methods. Samples ( $15-\mathrm{mm}$ in length) were obtained from the injured segment of spinal cord. Following dehydration in $25 \%$ sucrose at $4^{\circ} \mathrm{C}$, continuous longitudinal frozen sections ( $\sim 14-\mu \mathrm{m}$ in thickness) were prepared. The remaining rats in each group underwent the same procedure at 4 weeks. Following hematoxylin $(0.5 \%)$ and eosin $(1 \%)$ (H\&E) staining for $5 \mathrm{~min}$ at room temperature, the injured area of the spinal cord was observed under a light microscope. Observation and calculation of the damaged cavity area, the target volume area, and average area/section of regions of interest in the lesioned spinal cord were estimated from coronal sections using the Cavalieri method $(2,6)$.

Immunohistochemical staining. Selected frozen tissue sections of spinal cord injury (SCI) tissue at 2 weeks and 4 weeks following SCI underwent immunofluorescence staining. The sections were blocked with $20 \%$ donkey serum (Sigma-Aldrich; Merck KGaA) for $2 \mathrm{~h}$ at $37^{\circ} \mathrm{C}$ and washed in $0.01 \mathrm{M}$ PBS. The sections were then incubated with primary antibodies against the following in a refrigerator at $4^{\circ} \mathrm{C}$ overnight: Neurofilament (NF; N5264; 1:1,000), growth associated protein-43 (GAP-43; HPA013392; 1:1,000) and neuron-specific nuclear protein (NeuN; SAB4300883; dilution, 1:1,000) (all from Sigma-Aldrich; Merck KGaA). The

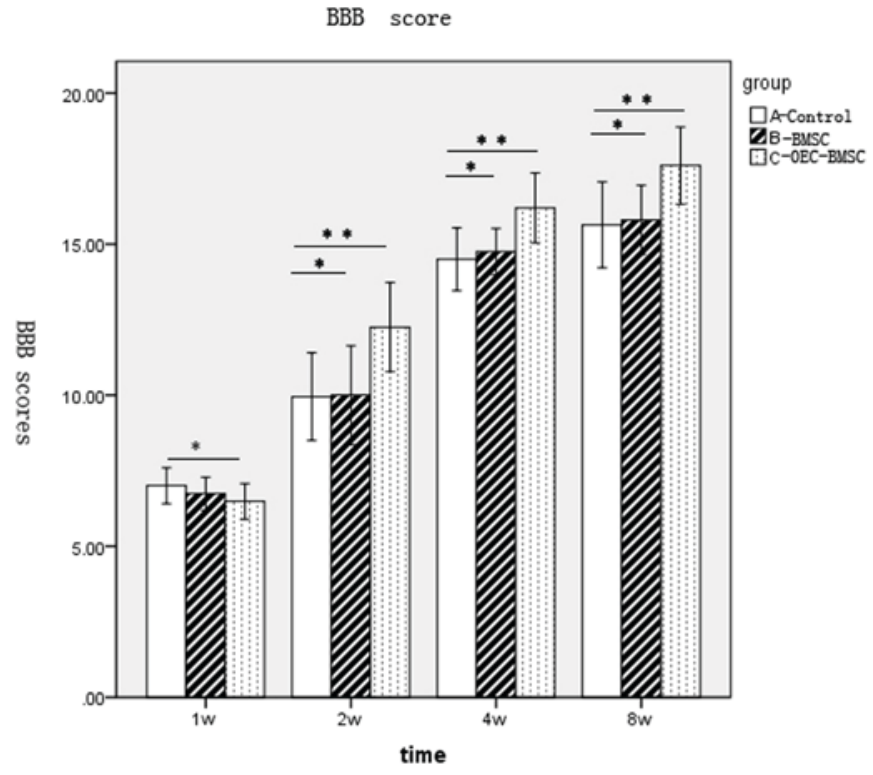

Figure 2. BBB score results of groups $\mathrm{A}, \mathrm{B}$ and $\mathrm{C}$ at each time point. " $\mathrm{P}>0.05$ and ${ }^{* *} \mathrm{P}<0.01$. BBB, Basso, Beattie and Bresnahan; w, week; group A, DMEM-treated control; group $\mathrm{B}$, normal BMSC group; group $\mathrm{C}$, olfactory ensheathing cell conditioned medium-induced BMSC group; BMSC, bone marrow stromal cell.

following day, the sections were washed 3 times with $0.01 \mathrm{M}$ PBS, and incubated for $24 \mathrm{~h}$ at $4^{\circ} \mathrm{C}$ with the following fluorescence-labeled secondary antibodies: fluorescein isothiocyanate (FITC)-labeled donkey anti-mouse IgG (A24501; 1:400) and Texas Red-labelled donkey anti-rabbit IgG (PA1-28590; 1:800) (both from Molecular Probes; Thermo Fisher Scientific, Inc.). The sections were then washed with PBS, mounted with glycerol and observed under a fluorescence microscope, where NF and GAP-43 double immunofluorescence was used. The relationship, similarity and difference in the expression of NF and GAP-43 were observed using confocal microscopy and analyzed using Adobe Photoshop CS2 (Adobe Systems, Inc., San Jose, CA, USA).

Statistical analysis. The resulting measurement data from all assays were normally distributed and expressed as the mean \pm standard deviation. Comparisons among multiple groups were performed using two-way analysis of variance. Student-Newman-Keuls was used as a post hoc test and the results between 4 and 8 weeks in each group was compared 


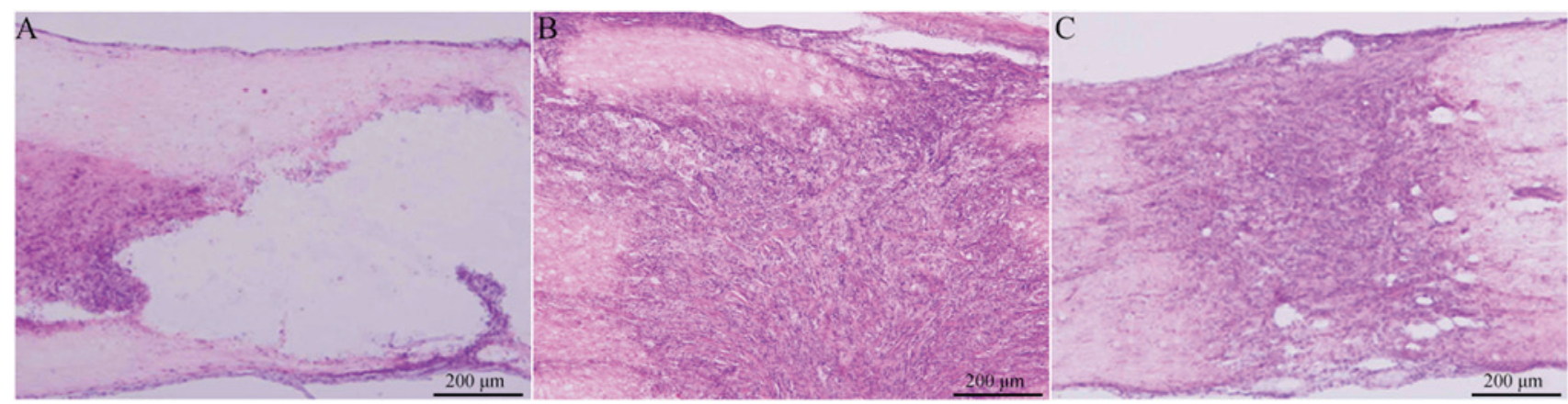

Figure 3. Spinal cord injury cavities of rats 2 weeks after injury in groups (A) A, (B) B, and (C) C, as determined by hematoxylin and eosin staining (magnification, x10). Group A, DMEM-treated control; group B, normal BMSC group; group C, olfactory ensheathing cell conditioned medium-induced BMSC group; BMSC, bone marrow stromal cell.

using the Student's t-test. SPSS statistical software (version, 19.0; IBM SPSS, Armonk, NY, USA) was used for data processing. $\mathrm{P}<0.05$ was considered to indicate a statistically significant difference.

\section{Results}

Primary culture of BMSCs and purification of OECs. BMSCs began to adhere and exhibit a stretched morphology at $48 \mathrm{~h}$ following inoculation (data not shown). The majority of cells exhibited fusiform growth, and cell colony formation was observed (data not shown). Following the medium change, cells began to proliferate rapidly, and when cells reached $80 \%$ confluence, fusion passage culture was performed. BMSCs were isolated by changing the medium to remove non-adherent cells in passage culture. In the third passage culture, the morphology of BMSCs was more consistent, demonstrating fusiform growth and strong refraction (Fig. 1A). Primary cultures of OECs were purified using the Nash differential adherent method and cytarabine pure culture. The growth state of the cells was observed under a light microscope. The majority of cells were bipolar and fusiform, most nuclei were oval and distributed in the center of cells, the cell bodies were clearly visible under the light microscope and the axons appeared elongated (Fig. 1B).

Transplantation of induced BMSCs improved motor function following spinal cord injury. The lower limbs of all rats with $\mathrm{SCI}$ by extrusion lost all motor function $24 \mathrm{~h}$ following injury and the muscle strength was 0 . The lowest score of motion in $\mathrm{BBB}$ ratings was 0 , while the maximum was 21 . The hind limb motor function of rats in group A appeared to recover at 1 week following surgery (Fig. 2). Functional recovery of behavior in each group was faster at 2-4 weeks following surgery, with the difference between BBB scores among groups increasing over time (Fig. 2). However, behavioral recovery in each group reduced during 4-8 weeks following surgery, and entered a relatively stable plateau. No significant difference in the BBB scores between groups B and A was observed; however, a significant difference between groups $\mathrm{C}$ and groups $\mathrm{A}$ and $\mathrm{B}$ was observed at 2, 4 and 8 weeks (Fig. 2).

Spinal cord injury area was reduced following transplantation of BMSCs. Cavity formation following spinal cord

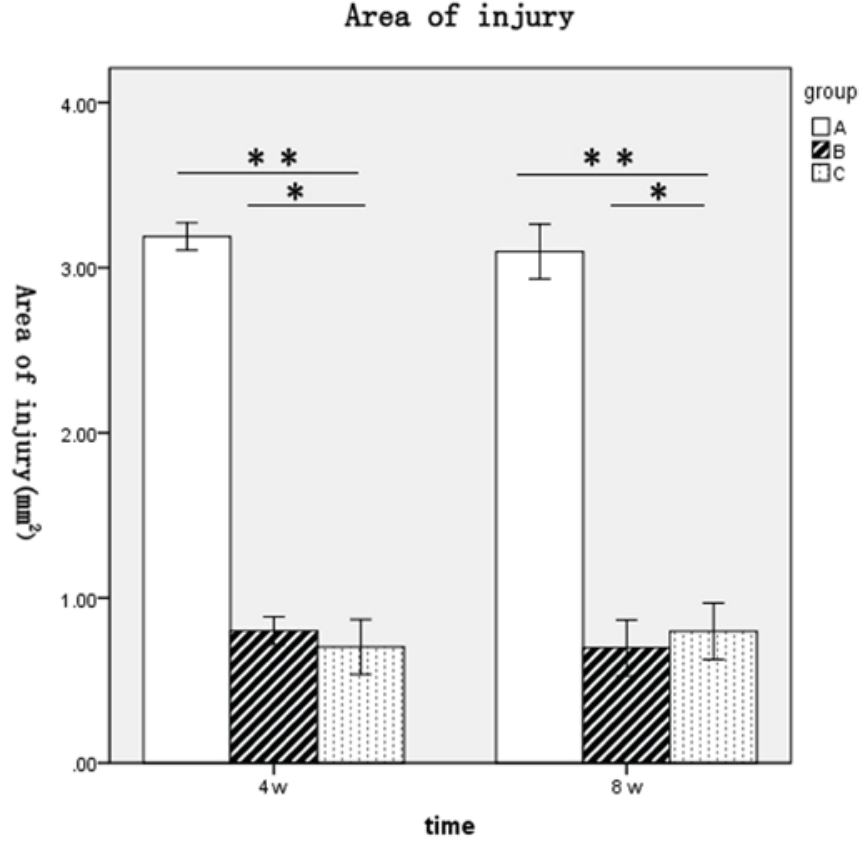

Figure 4. Comparison of spinal cord injury areas $\left(\mathrm{mm}^{2}\right)$ in groups A, B and $\mathrm{C}$ at 4 and 8 weeks following surgery. ${ }^{*} \mathrm{P}>0.05$ and ${ }^{* *} \mathrm{P}<0.01$. Group $\mathrm{A}$, DMEM-treated control; group B, normal BMSC group; group C, olfactory ensheathing cell conditioned medium-induced BMSC group; BMSC, bone marrow stromal cell.

injury was visualized by H\&E staining at 2 weeks following surgery. Frozen sections were stained with HE, observed under a light microscope and the cavity area was calculated. The cavity area of group A was visibly larger than the cavity area of group B or C, where proliferating BMSCs filled the damaged region, cell reproductive capacity was increased, and no evident cavity or necrotic cavity was detected (Fig. 3). The areas of spinal cord injury for rats in each group at 4 and 8 weeks following surgery were as follows: Group A, $3.19 \pm 0.1$ and $3.1 \pm 0.2 \mathrm{~mm}^{2}$, respectively; group $\mathrm{B}, 0.8 \pm 0.1$ and $0.7 \pm 0.2 \mathrm{~mm}^{2}$, respectively; and group $\mathrm{C}, 0.7 \pm 0.2$ and $0.8 \pm 0.2 \mathrm{~mm}^{2}$, respectively (Fig. 4). The spinal cord injury areas of rats in groups B and $\mathrm{C}$ were significantly lower when compared with group A at 4 and 8 weeks post-surgery $(\mathrm{P}<0.01$ and $\mathrm{P}<0.01$, respectively; Fig. 4). No significant difference between groups $\mathrm{B}$ and $\mathrm{C}$ at either time point was observed (Fig. 4). 


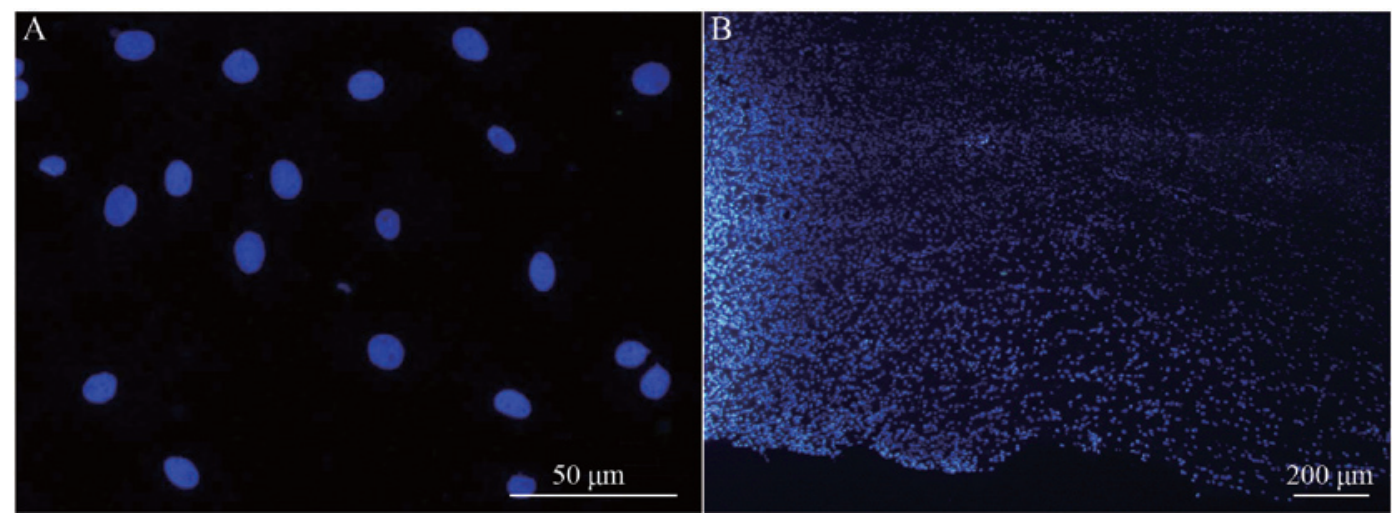

Figure 5. (A) Hoechst 33342-labeled BMSC nuclei before transplanting (magnification, x20), and (B) surviving and migrating BMSCs in the spinal cord injury area of a group B rat 2 weeks after injury. Light area to the left of the image is the injury center (magnification, x10). BMSC, bone marrow stromal cell.

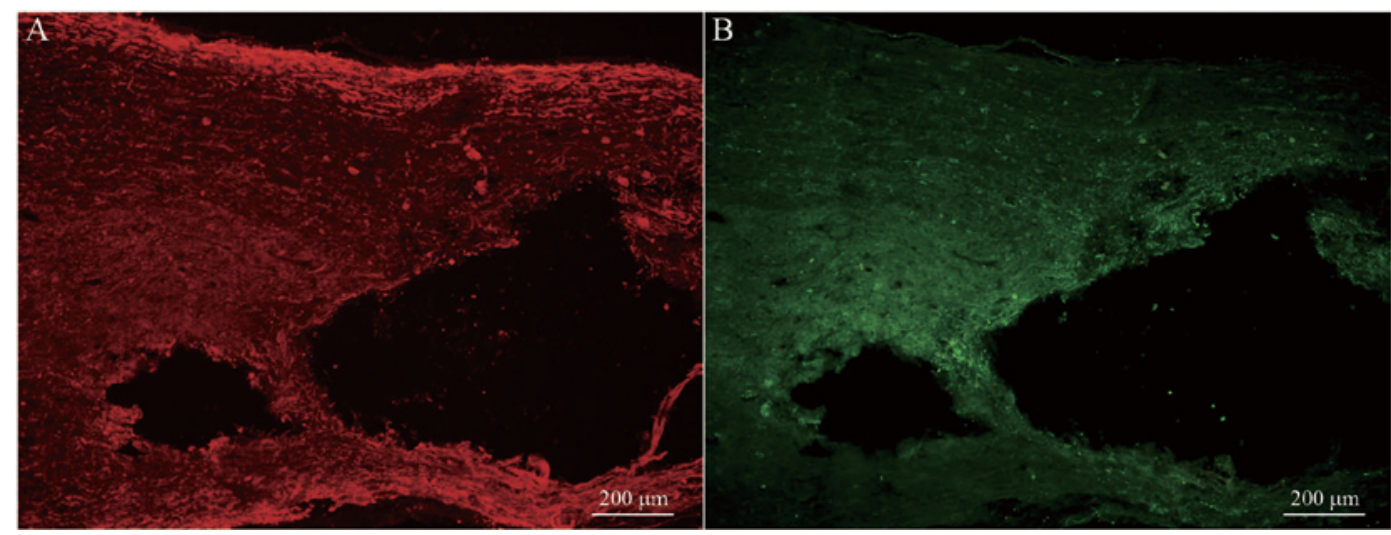

Figure 6. (A) Neurofilament and (B) growth associated protein-43 staining of the spinal cord injury area in the Dulbecco's modified Eagle's medium-treated control group of rats (group A; magnification, $\mathrm{x} 10$ ).
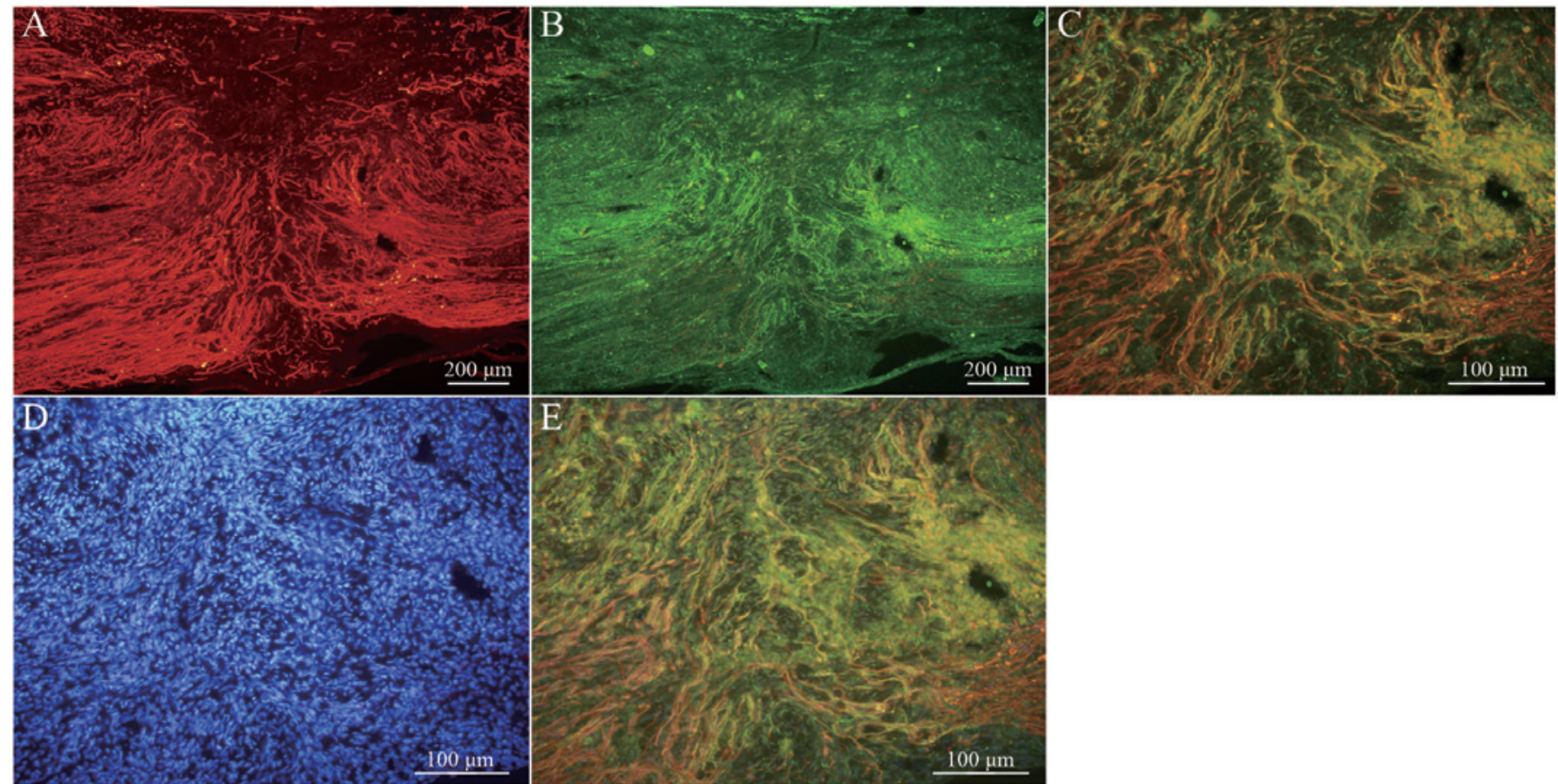

Figure 7. (A) NF and (B) GAP-43 staining of the spinal cord injury area in the olfactory ensheathing cell conditioned medium-induced BMSC group 4 weeks after injury (group C; magnification, x10). (C) Merged images of NF and GAP-43 dual-labeled immunofluorescence (magnification, x10). (D) Hoechst 33342-stained BMSC nuclei (magnification, x20). (E) Merged fluorescence image of NF, GAP-43 and Hoechst 33342 staining (magnification, x20). NF, neurofilament; GAP-43, growth associated protein-43; BMSC, bone marrow stromal cell. 

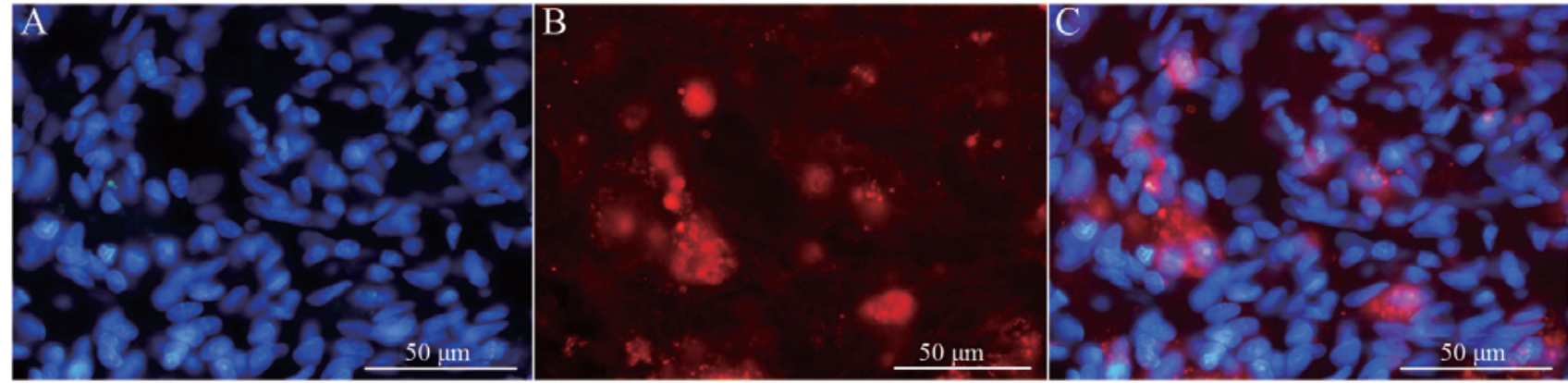

Figure 8. Staining of BMSCs cultured in olfactory ensheathing cell conditioned medium (group C) 2 weeks after injury with (A) Hoechst 33342 (magnification, $\mathrm{x} 40$ ) and (B) neuronal cell marker neuron-specific nuclear protein (magnification, x40). (C) Merged Hoechst 3342 and neuronal cell marker neuron-specific nuclear protein images (magnification, $\mathrm{x} 40$ ). BMSC, bone marrow stromal cell.
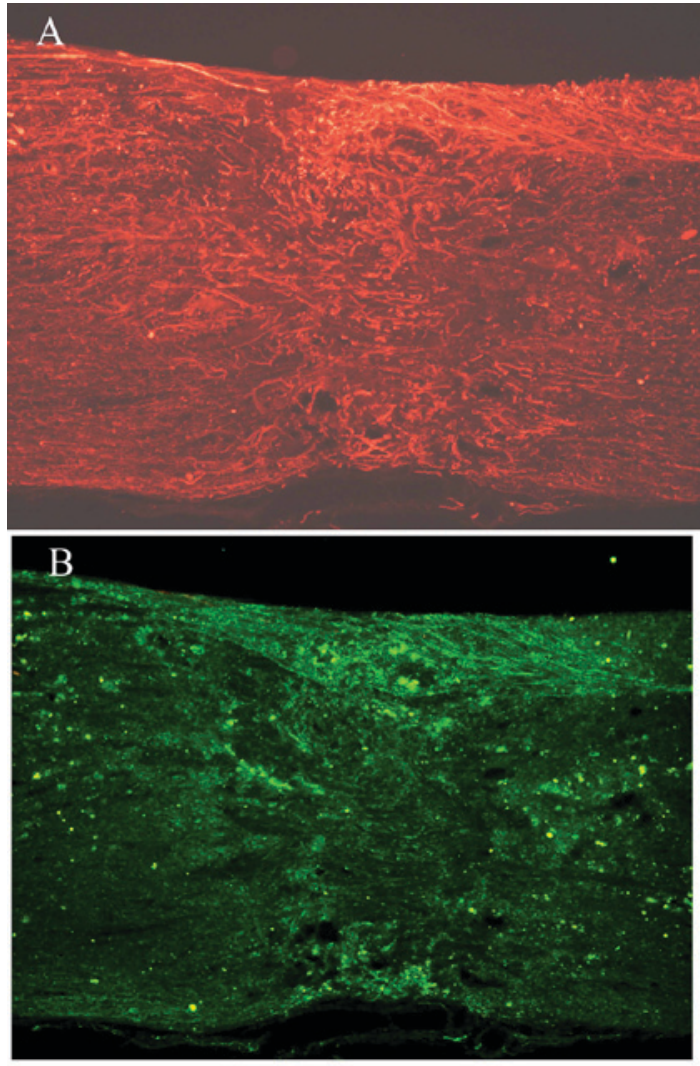

Figure 9. (A) NF and (B) GAP-43 staining of the spinal cord injury area in the BMSC group 4 weeks after injury (group B; magnification, x10). NF, neurofilament; GAP-43, growth associated protein-43; BMSC, bone marrow stromal cell.

Transplanted BMSCs survive and migrate in vivo. Longitudinal spinal cord sections from rats in groups $\mathrm{A}, \mathrm{B}$ and $\mathrm{C}$ were obtained at 2, 4 and 8 weeks following surgery, and fluorescence microscopy analysis revealed a large number of Hoechst 33342-labeled cells at the spinal cord injury site in groups B and $\mathrm{C}$. The majority of nuclei exhibited a soft uniform blue fluorescence, indicating that transplanted BMSCs survive in rats in vivo (Fig. 5A). The transplanted cells accumulated in the spinal cord transplantation sites, and migrated to the spinal cord parenchyma on both sides of the injury area (Fig. 5B).

Transplanted BMSCs differentiate into neuron-like cells in vivo. Immunofluorescence staining of NF and GAP-43 in

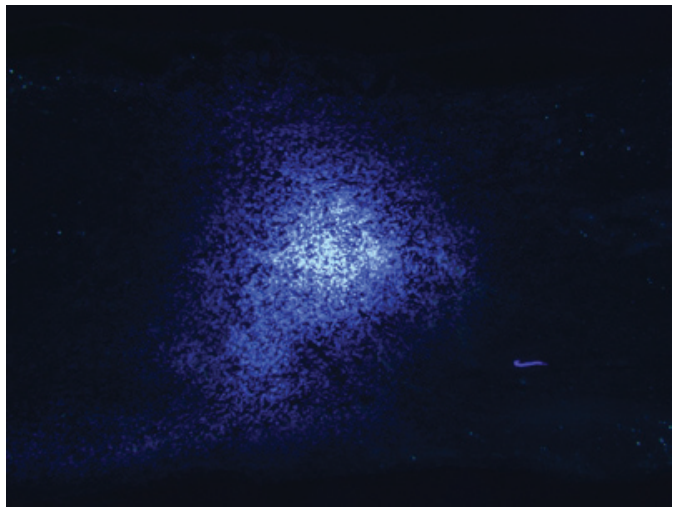

Figure 10. Surviving and migrating bone marrow stromal cells in the spinal cord injury area of a group B rat 2 weeks after injury. Light area is the injury center (magnification, x10).

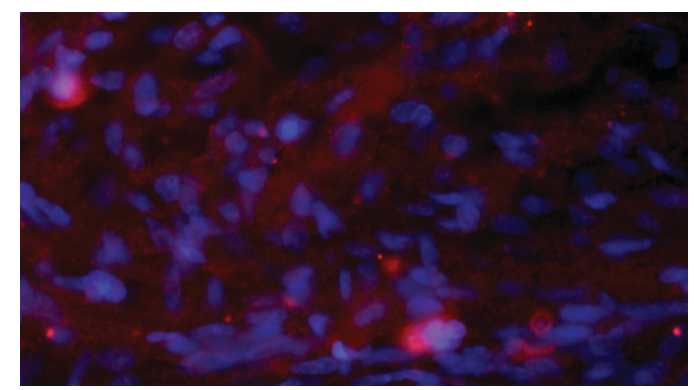

Figure 11. Merged Hoechst 33342 and neuronal cell marker neuron-specific nuclear protein images (group B) 2 weeks after injury (magnification, $\mathrm{x} 40$ ).

frozen tissue sections revealed that, while few NF or GAP-43 positive nerve fibers were observed in group A (Fig. 6A and B), continuous NF and GAP-43 immunoreactive nerve fibers in spinal cord injury sites were observed in group C (Fig. 7A and $\mathrm{B}$ ), which exhibited a consistent directional pattern of arrangement. NF and GAP-43 dual immunofluorescence results revealed that these proteins were distributed in the damaged area (Fig. 7C). In addition, Hoechst 33342-labeled BMSC nuclei aligned with the NF and GAP-43 dual immunofluorescence-stained neurons (Fig. 7D and E). This indicated that OEC conditioned medium-induced BMSCs promoted the regeneration of nerve fibers. In addition, when Hoechst 33342-labeled cells were transplanted in vivo (Fig. 8A) the expression of NeuN was partially positive (Fig. 8B and C), 
indicating that induced BMSCs are able to differentiate in vivo. Fig. 9 demonstrates NF and GAP-43 staining of the spinal cord injury area in the BMSC group 4 weeks after injury. Fig. 10 demonstrates surviving and migrating bone marrow stromal Hoechst 33342-labeled cells in the spinal cord injury area of a group B rat 2 weeks after injury and Fig. 11 is a merged Hoechst 33342 and neuronal cell marker neuron-specific nuclear protein images of a group B rat 2 weeks after injury.

\section{Discussion}

Complex alterations in the local microenvironment following spinal cord injury generate challenging conditions for efficient repair $(2,7)$. Investigating the application of cell transplantation for the treatment of spinal cord injury has been a recent focus of research. In the bone marrow stromal hematopoietic microenvironment, BMSCs are involved in supporting and regulating the process of bone marrow hematopoiesis. As specific stem cells, BMSCs are readily available, proliferate rapidly, exhibit weak immunogenicity and are autologous. Notably, BMSCs demonstrate a multi-differentiation ability $(28,29)$. Previous experimental studies have demonstrated that BMSCs differentiate into bone cells, muscle cells, neurons and glial cells under different induction conditions $(30,31)$. OECs are located in the olfactory bulb and the olfactory mucosa, and possess lifetime division potentials and regeneration characteristics. A previous study noted that OEC conditioned medium successfully induces BMSCs in vitro to differentiate into neural-like cells (32). In the present study, OEC conditioned medium-induced BMSCs were transplanted and used to treat spinal cord injury in a rat model. OEC conditioned medium-induced BMSCs transplantation was observed to significantly reduce cavity formation and the extent of damage following spinal cord injury. In addition, the induced BMSCs protected original nerve fibers of the spinal cord, promoted axonal regeneration and expressed the NeuN neuronal marker in vivo. Furthermore, these induced BMSCs improved lower extremity motor function in rats, indicating that OEC conditioned medium-induced BMSCs promoted repair of spinal cord injury in a certain context. However, the mechanisms underlying how these nerve fibers and cells established contact with the host in vivo, and performed their physiological functions requires further study.

In the present study, the functional recovery of the OEC conditioned medium-induced BMSC transplantation group was more apparent than in the normal BMSC transplantation group. This result may be due a number of factors. For instance, prior to transplantation, a large number of BMSCs in the OEC conditioned medium-induced group may be induced into neurons or nerve cells, and a higher number of nerve cells in the spinal cord injury site survived following transplantation, which provided an important basis for the regeneration and repair of spinal cord injury. In addition, prior to transplantation of OEC conditioned medium-induced BMSCs, undifferentiated BMSCs may have been able to proliferate rapidly following transplantation, thus reducing the damage caused by the formation of the cavity, which protected the host, transplanted nerve cells and nerve fibers, and provided dependent media for axonal regeneration. Furthermore, BMSCs have been previously demonstrated to secrete nutritional factors, including brain-derived neurotrophic factor and vascular endothelial growth factor, which may have protected neurons and promoted axonal regeneration in the present study (33). Finally, some undifferentiated BMSCs could differentiate into neurons in the spinal cord following transplantation, due to a variety of factors mentioned above (3). This provides an explanation as to why the lower extremity motor function recovery of rats in the OEC conditioned medium-induced BMSC transplantation group was improved when compared with the non-induced BMSC transplantation group.

In conclusion, the results of the present study demonstrated that OEC conditioned medium-induced BMSCs survived in vivo, reduced the formation of spinal cord injury cavities, promoted the regeneration of nerve fibers and enabled partial recovery of motor function in a rat model of spinal cord injury. However, although a greater number of axons were observed in the damaged region following transplantation of induced BMSCs, most of these axons were unordered (Fig. 7). The present study investigated the state of the transplanted BMSCs over the course of 8 weeks, and did not determine the effect of induced BMSC transplantation on the final outcome of spinal cord injury. Therefore, the present study is a limited primary investigation and further studies are required.

\section{Acknowledgements}

The present study was funded by the Hebei Medical Research Topic Program (grant no. 20150508).

\section{References}

1. Bonner JF and Steward O: Repair of spinal cord injury with neuronal relays: From fetal grafts to neural stem cells. Brain Res 1619: 115-123, 2015.

2. Taoka Y and Okajima K: Spinal cord injury in the rat. Prog Neurobiol 56: 341-358, 1998.

3. Ankeny DP, McTigue DM and Jakeman LB: Bone marrow transplants provide tissue protection and directional guidance for axons after contusive spinal cord injury in rats. Exp Neurol 190: 17-31, 2004.

4. Qiu XC, Jin H, Zhang RY, Ding Y, Zeng X, Lai BQ, Ling EA, Wu JL and Zeng YS: Donor mesenchymal stem cell-derived neural-like cells transdifferentiate into myelin-forming cells and promote axon regeneration in rat spinal cord transaction. Stem Cell Res Ther 6: 105, 2015.

5. Collyer E, Catenaccio A, Lemaitre D, Diaz P, Valenzuela V, Bronfman F and Court FA: Sprouting of axonal collaterals after spinal cord injury is prevented by delayed axonal degeneration. Exp Neurol 261: 451-461, 2014.

6. Plunet WT, Streijger F, Lam CK, Lee JH, Liu J and Tetzlaff W: Dietary restriction started after spinal cord injury improves functional recovery. Exp Neurol 213: 28-35, 2008.

7. Slotkin JR, Pritchard CD, Luque B, Ye J, Layer RT, Lawrence MS, O'Shea TM, Roy RR, Zhong H, Vollenweider I, et al: Biodegradable scaffolds promote tissue remodeling and functional improvement in non-human primates with acute spinal cord injury. Biomaterials 123: 63-76, 2017.

8. Deng LX, Walker C and Xu XM: Schwann cell transplantation and descending propriospinal regeneration after spinal cord injury. Brain Res 1619: 104-114, 2015.

9. Nakano N, Nakai Y, Seo TB, Homma T, Yamada Y, Ohta M, Suzuki Y, Nakatani T, Fukushima M, Hayashibe M and Ide C: Effects of bone marrow stromal cell transplantation through CSF on the subacute and chronic spinal cord injury in rats. PLoS One 8: e73494, 2013.

10. Tharion G, Indirani K, Durai M, Meenakshi M, Devasahayam SR, Prabhav NR, Solomons C and Bhattacharji S: Motor recovery following olfactory ensheathing cell transplantation in rats with spinal cord injury. Neurol India 59: 566-572, 2011. 
11. Wright KT, Uchida K, Bara JJ, Roberts S, El Masri W and Johnson WE: Spinal motor neurite outgrowth over glial scar inhibitors is enhanced by coculture with bone marrow stromal cells. Spine J 14: 1722-1733, 2014.

12. Sun T, Ye C, Zhang Z, Wu J and Huang H: Cotransplantation of olfactory ensheathing cells and Schwann cells combined with treadmill training promotes functional recovery in rats with contused spinal cords. Cell Transplant 22 (Suppl 1): S27-S38, 2013.

13. Tropel P, Platet N, Platel JC, Noël D, Albrieux M, Benabid AL and Berger F: Functional neuronal differentiation of bone marrow-derived mesenchymal stem cells. Stem Cells 24: 2868-2876, 2006.

14. Liu Z, He B, Zhang RY, Zhang K, Ding Y, Ruan JW, Ling EA, $\mathrm{Wu} \mathrm{JL}$ and Zeng YS: Electroacupuncture promotes the differentiation of transplanted bone marrow mesenchymal stem cells pre-induced with neurotrophin-3 and retinoic acid into oligodendrocyte-like cells in demyelinated spinal cord of rats. Cell Transplant 24: 1265-1281, 2015.

15. Akiyama Y, Radtke C and Kocsis JD: Remyelination of the rat spinal cord by transplantation of identified bone marrow stromal cells. J Neurosci 22: 6623-6630, 2002.

16. Woodbury D, Schwarz EJ, Prockop DJ and Black IB: Adult rat and human bone marrow stromal cells differentiate into neurons. J Neurosci Res 61: 364-370, 2000.

17. Barzilay R, Melamed E and Offen D: Introducing transcription factors to multipotent mesenchymal stem cells: Making transdifferentiation possible. Stem Cells 27: 2509-2515, 2009.

18. Ni WF, Yin LH, Lu J, Xu HZ, Chi YL, Wu JB and Zhang N: In vitro neural differentiation of bone marrow stromal cells induced by cocultured olfactory ensheathing cells. Neurosci Lett 475: 99-103, 2010.

19. Yui S, Fujita N, Chung CS, Morita M and Nishimura R: Olfactory ensheathing cells (OECs) degrade neurocan in injured spinal cord by secreting matrix metalloproteinase- 2 in a rat contusion model. Jpn J Vet Res 62: 151-162, 2014.

20. Ninomiya K, Iwatsuki K, Ohnishi $Y$, Ohkawa $T$ and Yoshimine T: Intranasal delivery of bone marrow stromal cells to spinal cord lesions. J Neurosurg Spine 23: 111-119, 2015.

21. Torres-Espín A, Redondo-Castro E, Hernández J and Navarro X: Bone marrow mesenchymal stromal cells and olfactory ensheathing cells transplantation after spinal cord injury-a morphological and functional comparison in rats. Eur J Neurosci 39: 1704-1717, 2014.
22. Kastenmayer RJ, Moore RM, Bright AL, Torres-Cruz R and Elkins WR: Select agent and toxin regulations: Beyond the eighth edition of the guide for the care and use of laboratory animals. J Am Assoc Lab Anim Sci 51: 333-338, 2012.

23. Nash HH, Borke RC and Anders JJ: New method of purification for establishing primary cultures of ensheathing cells from the adult olfactory bulb. Glia 34: 81-87, 2001.

24. Balao da Silva C, Macías-García B, Morillo Rodriguez A, Gallardo Bolaños JM, Tapia JA, Aparicio IM, Morrell JM, Rodriguez-Martínez H, Ortega-Ferrusola C and Peña FJ: Effect of Hoechst 33342 on stallion spermatozoa incubated in KMT or Tyrodes modified INRA96. Anim Reprod Sci 131: 165-171, 2012.

25. Sedý J, Urdzíková L, Jendelová P and Syková E: Methods for behavioral testing of spinal cord injured rats. Neurosci Biobehav Rev 32: 550-580, 2008.

26. Howard CV and Reed MG: Unbiased stereology: Threedimensional measurement in microscopy. J Anat 194: 153-157, 1999.

27. Vawda R, Soubeyrand M, Zuccato JA and Fehlings MG: Spinal cord injury and regeneration: A critical evaluation of current and future therapeutic strategies. Pathobiol Hum Dis 8: 593-638, 2014.

28. Xu Y, Xiong F, Liu L and Zhang C: Rat bone marrow stromal cells could be induced into Schwann cell precursor-like cells in vitro. Neurosci Lett 488: 229-233, 2011.

29. Robey PG, Kuznetsov SA, Ren J, Klein HG, Sabatino M and Stroncek DF: Generation of clinical grade human bone marrow stromal cells for use in bone regeneration. Bone 70: 87-92, 2015.

30. Chiu LH, Lai WF, Chang SF, Wong CC, Fan CY, Fang CL and Tsai YH: The effect of type II collagen on MSC osteogenic differentiation and bone defect repair. Biomaterials 35: 2680-2691, 2014.

31. Sanchez-Ramos J, Song S, Cardozo-Pelaez F, Hazzi C, Stedeford T, Willing A, Freeman TB, Saporta S, Janssen W, Patel N, et al: Adult bone marrow stromal cells differentiate into neural cells in vitro original research article. Exp Neurol 164: 247-256, 2000.

32. Ni WF, Yin LH, Lu J, Xu HZ, Chi YL, Wu JB and Zhang N: In vitro neural differentiation of bone marrow stromal cells induced by cocultured olfactory ensheathing cells. Neurosci Lett 475: 99-103, 2010.

33. Neuhuber B, Timothy Himes B, Shumsky JS, Gallo G and Fischer I: Axon growth and recovery of function supported by human bone marrow stromal cells in the injured spinal cord exhibit donor variations. Brain Res 1035: 73-85, 2005. 